\title{
La competencia en alimentación. Un marco de referencia para la educación obligatoria
}

\section{A framework for developing food competence in compulsory education}

\author{
Enrique Espańa Ramos \\ enrienri@uma.es \\ Aurelio Cabello Garrido \\ aacabellogarrido@gmail.com \\ Ángel Blanco López \\ ablancol@uma.es \\ Universidad de Málaga, Didáctica de las Ciencias Experimentales
}

RESUMEN • En este artículo se presenta un marco teórico de referencia para desarrollar la competencia en alimentación en la educación obligatoria. Los planteamientos seguidos para su elaboración implican salir inicialmente del contexto escolar y situarse en la vida diaria para conocer cuál es la situación actual en nuestra sociedad. De esta forma se puede resaltar qué aspectos son los más necesarios y en cuáles se detectan mayores carencias, para incidir especialmente en ellos. A partir de la definición y delimitación del contexto se caracteriza la competencia en alimentación que un ciudadano debería poseer. Como resultado se describe el marco de referencia para su desarrollo en la educación obligatoria, organizado en torno a siete dimensiones: los alimentos, el funcionamiento del cuerpo con respecto a la nutrición, cocinar, cultivar y elaborar los alimentos, comprar alimentos, comer en compañía, la actividad física y el descanso.

PALABRAS CLAVE: enseñanza en contexto; competencia en alimentación; educación obligatoria.

ABSTRACT • In this paper, a theoretical framework for developing Food Competence in compulsory education is presented. The approaches involved initially lead us to leave the school context and move into daily life in order to find out what the current situation in our society is. Thus, it was possible to focus on those aspects which are more necessary and which are found in greatest need with a view to act on them specifically. Based on the definition of human nutrition and the limits of this context, the Food Competences that a citizen should have are characterized in this paper. As a result, the framework for its development in compulsory education is described. It has been organized around seven axes: food, the body's work regarding nutrition, cooking, growing and processing food, buying groceries, eating with others, physical activity and rest.

KEYWORDS: context-based; food competence; compulsory education.

Fecha de recepción: enero 2013 • Aceptado: julio 2013

España, E., Cabello, A., Blanco, A. (2014) La competencia en alimentación. Un marco de referencia para la educación obligatoria Enseñanza de las Ciencias, 32.3, pp. 611-629 


\section{INTRODUCCIÓN}

Hoy día existe un notable consenso en que la mejora de la alfabetización científica de los ciudadanos debe constituir la finalidad de la educación científica (Bybee, 1997; Fourez, 1997; Banet, 2010). La alfabetización científica se propone formar a ciudadanos capaces de tomar decisiones responsables en asuntos que les afectan en sus vidas y que están relacionados con la ciencia y la tecnología. A pesar de este amplio consenso todavía no puede decirse, por muy diversas razones, que este concepto impregne la práctica en la enseñanza de las ciencias de forma significativa (Fensham, 2007; Banet, 2010).

Existe una gran distancia entre lo que se enseña habitualmente en las aulas y la vida diaria (Hernández, 2006), hasta el punto de que muchos estudiantes tienen serias dificultades para encontrar sentido a lo que se les enseña (Sanmartí, Burgoa y Nuño, 2011). Y ello sucede a pesar de que la necesidad de dar sentido a lo que se aprende es una cuestión ampliamente aceptada en el ámbito de la investigación educativa en general (Pérez Gómez, 2008), y en el de la didáctica de las ciencias en particular, no solo por razones didácticas, sino también epistemológicas, ya que los conceptos científicos surgen de situaciones problemáticas y, por lo tanto, requieren una situación real en la que se apliquen y en la que adquieran sentido (Chamizo e Izquierdo, 2005).

Para ayudar al estudiante a otorgar sentido a lo que aprende se ha propuesto contextualizar la ciencia que se enseña (Caamaño, 2005) o construir la ciencia escolar a partir de necesidades contextualizadas (Pro y Rodríguez, 2010). En términos generales podemos decir que se trata de relacionar, de forma significativa, la ciencia con la vida diaria de los estudiantes y poner de manifiesto su relevancia en los ámbitos personal, profesional y social.

Aunque la propuesta de utilizar los contextos en la enseñanza de las ciencias no es, ni mucho menos, una cuestión novedosa, la introducción de las competencias básicas en los currículos de la educación obligatoria y, sobre todo, en el programa PISA (OCDE, 2006) ha situado de nuevo en el centro de atención la necesidad de tener en cuenta los contextos en la enseñanza (Sanmartí, Burgoa y Nuño, 2011), y quizás, como algo más novedoso, en la evaluación (Fensham, 2009). PISA 2006 incluye un apartado dedicado a situaciones y contexto, en el que define la situación como la parte del universo del estudiante en que se sitúan las tareas que se han de realizar. En las unidades de evaluación se utilizan fundamentalmente situaciones centradas en cinco grandes ámbitos: salud, recursos naturales, medio ambiente, riesgos y fronteras de la ciencia y la tecnología, abordados a través de tres niveles de relevancia: personal, social y global (Fensham, 2009).

Existen diversas formas de entender el concepto de contexto (Gilbert, 2006) y el papel que debe desempeñar en el proceso de enseńanza y aprendizaje (De Jong, 2006). En unos casos se utiliza como ilustración o aplicación después de haber enseñado los conceptos. En otros casos, se parte del contexto como orientación o motivación inicial para después desarrollar los conceptos. En los planteamientos más actuales se entiende el contexto como una situación o problema complejo, relevante socialmente y del entorno del alumnado (Gilbert, 2006; Sanmartí, Burgoa y Nuño, 2011), que sirve de eje vertebrador del currículo y de la enseñanza (De Jong, 2006). Las situaciones del mundo real planteadas como contextos incluyen problemas que pueden afectarnos a tres niveles (Gutiérrez, 2006): como individuos (por ejemplo, la alimentación, la sexualidad, la práctica deportiva o el empleo de la energía), como miembros de una comunidad local (por ejemplo, el tratamiento del agua o la ubicación de una central eléctrica) o como ciudadanos del mundo (por ejemplo, el calentamiento global o la disminución de la biodiversidad). Todas estas cuestiones demandan un análisis sosegado y una toma personal de decisiones, en las que tienen un papel importante los conocimientos científicos, las habilidades, las actitudes, los valores, la disposición para la acción, etc. 
El desarrollo de competencias básicas en la educación obligatoria (MEC, 2006) y, concretamente, de la competencia científica se presenta como una oportunidad de llevar a la práctica propuestas que integren los problemas del contexto de la vida diaria en las clases de ciencias (Pro, 2012).

Una tarea previa importante, antes de diseñar este tipo de propuestas didácticas, consistiría en identificar situaciones que se consideren relevantes para los ciudadanos, con un valor educativo per se (Perrenoud, 2012) y cuyo tratamiento en el aula permita el desarrollo de la competencia científica y otras competencias básicas. Para ello se ha considerado útil salir del contexto escolar y situarnos en la vida diaria para conocer cuál es la situación actual en nuestra sociedad, y así poder resaltar qué aspectos son los más necesarios para la vida (Perrenoud, 2012).

Ahora bien, no se trata, ni muchos menos, de una empresa simple. En primer lugar, por la dificultad que supone determinar cuáles son las necesidades, los problemas y las situaciones relevantes que cualquier persona deberá afrontar a lo largo de la vida. Como indica Perrenoud (2012),

... lo más razonable es pensar que algunas situaciones seguirán siendo bastante similares a las que conocemos y que otras serán radicalmente nuevas, en concreto en las áreas donde las tecnologías, y sobre todo las biotecnologías, van a cambiar la propia vida, la relación con la enfermedad y la muerte, el espacio, el tiempo, el pensamiento, el trabajo, la familia, la delincuencia, el aprendizaje... (p. 114).

En segundo lugar, suponiendo que se hayan identificado las competencias necesarias para afrontar las necesidades, problemas y situaciones relevantes de la vida diaria, habrá que distinguir aquello que es responsabilidad exclusiva de la educación formal (el sistema escolar) de aquellos otros aspectos en los que su función es de corresponsabilidad, o solo de complementariedad, con respecto a la educación informal (la familia, los medios de comunicación y las relaciones sociales) y la educación no formal (como museos o centros de ciencia y tecnología).

En el proyecto de investigación (Blanco, España y González, 2010) en el que se enmarca este trabajo se han considerado los siguientes contextos, teniendo en cuenta su relevancia para escolares del rango de edades considerados en este (de 10 a 16 años): alimentación, actividad física y salud, sexualidad; medio ambiente y sostenibilidad, y medios de comunicación y problemas derivados del consumo de drogas. Se trata de tres grandes ámbitos importantes de la vida de los ciudadanos: la salud, el medio ambiente y la comunicación. Aunque consideramos que estos contextos tienen suficiente relevancia, no hemos pretendido abarcar todos los que serían necesarios desarrollar en la educación obligatoria.

En este artículo nos centramos en la alimentación, por tratarse de un contexto con gran repercusión en diferentes aspectos de la vida diaria de las personas, especialmente en el ámbito de la salud (Merelles et al., 2005), pero también en el de las relaciones sociales o en el de la economía individual y familiar. Así mismo, se trata de un dominio específico, incluido en los currículos escolares, que ha sido objeto de gran atención en la investigación didáctica (por ejemplo, Banet, 2001; Cabello, Blanco y España, 2009) y en el desarrollo curricular (por ejemplo, Banet y Núñez, 1992; Membiela y Cid, 1998; García y Martínez, 2009).

En concreto, el objetivo de este artículo es plantear un marco de referencia para desarrollar la competencia en alimentación en la educación obligatoria. Para ello, a partir de una revisión actualizada de estudios sobre el tema, se delimita y se define qué se entiende por contexto de la alimentación y se analizan los problemas detectados en la población infantil y juvenil española en relación con este contexto. A continuación, se lleva a cabo la caracterización de la competencia en alimentación. Finalmente, se describen y se analizan las dimensiones de la competencia en alimentación que se proponen para su desarrollo durante la educación obligatoria. 


\section{EL CONTEXTO DE LA ALIMENTACIÓN}

La alimentación puede entenderse como el proceso por el que una persona selecciona los alimentos que han de configurar su dieta y los prepara para su ingestión (Salvador y Serra, 1993). Se trata de un proceso voluntario y consciente, aunque limitado por la disponibilidad de alimentos, por los recursos económicos y por los hábitos culturales. Así pues, depende de una decisión individual y, por lo tanto, es educable, si bien los hábitos de cada persona constituyen un elemento muy resistente a cualquier cambio. Merelles et al. (2005) lo consideran un proceso fundamentalmente social.

La nutrición, por el contrario, tiene lugar a nivel celular y constituye un conjunto de procesos automáticos, involuntarios y continuos, a través de los cuales el organismo aprovecha las sustancias contenidas en los alimentos ingeridos (Salvador y Serra, 1993). La nutrición, lógicamente, es inconsciente y, por lo tanto, no es educable. Solo una buena alimentación puede asegurar un estado nutricional adecuado. Para Merelles et al. (2005) es un proceso estrictamente biológico.

Según Gargallo (1998), la alimentación de un individuo concreto es equilibrada si alcanza los requerimientos de energía y de cada uno de los nutrientes esenciales, sin conducir a excesos injustificados, y, a la vez, le permite mantener un adecuado estado de salud y le capacita para realizar el ejercicio que exige cada tipo de trabajo, teniendo en cuenta su edad y su situación fisiológica. Puesto que no existe un tipo de alimento que por sí solo contenga la cantidad adecuada de estos nutrientes, el citado equilibrio se alcanzará combinando distintos tipos de alimentos.

La demostrada interrelación entre alimentación y nutrición transforma a ambos procesos en un aspecto prioritario de la salud pública, por lo que la alimentación puede considerarse el factor más importante de la higiene individual, ya que ningún otro factor aislado ejerce tanta influencia sobre la salud y sobre la vida (Merelles et al., 2005) o el factor ecológico-social externo más importante para la salud, tanto para el individuo como para la población (San Martín, 1989).

La selección de alimentos y la forma de prepararlos y manipularlos se ven poderosamente influidas por factores culturales y socioeconómicos. La tradición, la moda, las creencias religiosas y las preferencias individuales, especialmente de la persona que se encarga de alimentar a la familia, son los principales elementos culturales que condicionan la alimentación (Merelles et al., 2005).

Las grandes empresas tratan de modificar las preferencias individuales a través de la publicidad y de los medios de comunicación. Para Wilkinson y Marmot (2003), la alimentación saludable es una cuestión política en la medida en que las fuerzas del mercado mundial controlan el suministro de alimentos. Las condiciones sociales impuestas por las industrias alimentarias, favorecidas por los acuerdos comerciales internacionales (Acuerdo General sobre Aranceles Aduaneros y Comercio, entre otros), perjudican finalmente a los consumidores de menor poder adquisitivo, que tienden a sustituir los alimentos frescos por la comida procesada barata producida por las empresas que dominan el mercado. Estos autores consideran esta situación un claro ejemplo de gradiente o pendiente social en salud, concepto acuñado en los clásicos estudios Whitehall I y II (Marmot et al., 1991), que evidencian cómo la organización social afecta a la salud de las personas: la morbilidad o mortalidad por cualquier causa es mayor conforme bajamos en el nivel socioeconómico.

La pendiente social en la calidad de la dieta está relacionada con la denominada transición nutricional (Drewnowski y Popkin, 1997), escenario definido por el aumento del consumo de alimentos altamente procesados, grasos y azucarados, de elevada densidad energética, en comparación con las dietas tradicionales, caracterizadas por un mayor consumo de cereales poco procesados y de alimentos frescos. Las últimas estadísticas sanitarias mundiales (oms, 2012) ponen de relieve el creciente problema que representan las llamadas enfermedades no transmisibles (ENT). Muchas de ellas, como la diabetes y la hipertensión, están relacionadas con la alimentación. En las tres últimas décadas, de intensa globalización económica, el número de personas con sobrepeso/obesidad, y aquellas con enfermedades crónicas 
relacionadas con la dieta, ha aumentado de manera significativa. El incremento ha sido particularmente rápido en los países en desarrollo y más patente en los grupos de menor nivel socioeconómico (Hawkes, 2007), de acuerdo con el modelo propuesto por Wilkinson y Marmot (2003) a través de los determinantes sociales de la salud. Por lo tanto, la lucha contra la obesidad se ha convertido en uno de los mayores retos de la salud pública para el siglo XXI en todo el mundo.

En nuestro país, la transición nutricional se traduce en el progresivo abandono de la dieta mediterránea tradicional, al que estamos asistiendo desde hace años (García-Closas, Berenguer y González, 2006; da Silva et al., 2009), lo que también está conduciendo a un aumento de las ENT. Las estadísticas citadas indican que en el 2008 el 24,9\% de los españoles de más de 20 años y el 23\% de las españolas de la misma edad presentaban obesidad. Los resultados de otras investigaciones confirman estos datos: la población española con sobrepeso (IMC $>25 \mathrm{~kg} / \mathrm{m}^{2}$ ) era del 52,60\%, según la Encuesta Nacional de Salud (INE, 2006); del 53,65\%, según la EHIs-2009 (IIs, 2011), y del 61,30\%, según el Estudio ENRICA (Banegas et al., 2011).

Entre los jóvenes, el problema es preocupante. Serra y Aranceta (“Estudio enKid”, 2000) hallaron que el porcentaje de niños y jóvenes españoles con exceso de peso era del 26,3\% (Serra et al., 2003). En el Estudio AVEna el grupo con sobrepeso se situaba en el 25,69\% de los varones y el 19,13\% de las mujeres (Wärnberg et al., 2006). Finalmente, los resultados preliminares del Estudio ALAdino (Ministerio de Sanidad, 2011) establecieron en un 30,8\% el exceso de peso entre niños/as y jóvenes españoles, si bien aplicando los nuevos estándares de crecimiento de la oms este porcentaje alcanzaba el 44,5\% de la población. Según las investigaciones, esta situación es producto de la confluencia de dos comportamientos: escasa actividad física y dieta inadecuada.

Por un lado, los adolescentes españoles llevan un estilo de vida sedentario, dedicando demasiado tiempo a actividades desarrolladas frente a pantallas. Un adolescente promedio podría estar más de cuatro horas y media al día frente a una pantalla, es decir, el doble del máximo tolerable, mientras que el $40 \%$ de ellos no realiza ningún tipo de actividad física vigorosa al menos dos o tres días a la semana (Moreno et al., 2008). El Estudio aladino (Ministerio de Sanidad, 2011) ha encontrado una correlación inversa entre el tiempo pasado los fines de semana frente a un televisor, una consola de juegos o un reproductor de discos DVD y la prevalencia de sobrepeso. Los adolescentes espańoles mostraron menor fuerza muscular y peor capacidad aeróbica que otros adolescentes europeos y un $20 \%$ de ellos tiene riesgo elevado de padecer en el futuro una enfermedad cardiovascular (Ortega $e t$ al., 2005).

Este estilo de vida sedentario, con ocio pasivo, desemboca en un descanso nocturno insuficiente, que, junto a otras circunstancias, lleva a que el desayuno de muchos de nuestros jóvenes no sea adecuado. Más recientemente se ha empezado a vislumbrar que la privación crónica del sueño puede influir en el peso a través de efectos sobre el apetito, la actividad física y/o la termorregulación (Patel y Hu, 2008). Con un desayuno completo (compuesto de fruta, un alimento lácteo y cereales) se reduce la necesidad de consumir alimentos menos nutritivos a media mañana y se mejora el rendimiento físico e intelectual en clase. Sin embargo, apenas un 5\% lo lleva a cabo (Serra y Aranceta, 2000). Solo un 61\% de los adolescentes españoles desayuna regularmente los siete días de la semana (Moreno et al., 2005). Los adolescentes desayunan cada vez menos conforme se hacen mayores, sobre todo las chicas, siendo los de capacidad adquisitiva alta quienes más días a la semana desayunan (Ramos, 2009).

Por otra parte, su dieta habitual, al igual que la de los adultos, suele incluir una excesiva cantidad de alimentos de origen animal, en perjuicio de los de origen vegetal, lo que conduce a un perfil calórico alejado del recomendado, tanto para la población general como la juvenil. El perfil recomendado es el propio de la dieta mediterránea tradicional: un $60 \%$ de las calorías ingeridas deben proceder de los carbohidratos, un 30\% de los lípidos y un 10\% han de ser aportadas por las proteínas (Varela, Moreiras y Carbajal, 1988). El perfil calórico de la población española es: carbohidratos, 42\%; lípidos, 
40\%; proteínas, 14\% (del Pozo et al., 2012). El de los jóvenes es 44-40-16\%, incluyendo una ingesta energética diaria mayor que la recomendada (Fernández San Juan, 2006).

Como consecuencia, la dieta de los españoles es deficitaria en fibra, en algunas vitaminas y en algunos minerales. Según del Pozo et al. (2012), se ingieren 19,7 g/día de fibra, mucho menos que los más de $25 \mathrm{~g} /$ día recomendados. Esto podría explicar que cerca del $20 \%$ de españoles presente estreñimiento (Polanco et al., 2007). El consumo de fibra dietética entre los adolescentes españoles es también deficitario (Warnberg et al., 2006).

En cuanto a las vitaminas, del Pozo et al. (2012) han encontrado, entre la población general, ingestas inadecuadas para el folato y la vitamina D (esta solo para el grupo de edad de 50 a 59 ańos). En la población juvenil, Serra et al. (2001) observaron ingestas insuficientes de vitaminas A, C y E en niños varones. En los jóvenes, destacaron bajos consumos de vitaminas E, A y (solo en las chicas) de folato.

Con respecto a los minerales, del Pozo et al. (2012) han hallado tres problemas principales: existe un riesgo de ingesta insuficiente de cinc; la relación entre calcio y fósforo presenta un valor inferior a 1 , y el consumo de potasio no llega al valor deseado en los objetivos nutricionales (Moreiras et al., 2010). En cuanto a los jóvenes, Fernández-San Juan (2006) indicó que la ingesta media de magnesio, hierro, calcio y cinc estaba por debajo de las referencias.

Junto a estas carencias, la ingesta de sal de los españoles es muy alta: 9,8 gramos por persona y día, el doble de lo recomendado (Ortega et al., 2011). Asimismo, las bebidas refrescantes se consumen en exceso (del Pozo et al., 2012), sobre todo en las familias con menos recursos, que son, al mismo tiempo, las que menos frutas y verduras consumen.

Diferentes estudios han puesto de manifiesto que existen otros aspectos no relacionados directamente con la ingesta que tienen una gran repercusión en los hábitos alimentarios de las personas. En la última década, muchos autores han vinculado la capacidad para cocinar con el consumo de comidas más saludables (Larson et al., 2006), con la compra de más frutas y verduras (Winkler y Turrell, 2009) y con un menor uso de alimentos precocinados (Short, 2003) y de restaurantes de comida rápida (Soliah, Walter y Antosh, 2006). Los niños que comen junto a otros miembros de su familia consumen más frutas y verduras y menos grasas trans y saturadas, beben menos refrescos y presentan una carga glucémica menor que quienes comen solos frente al televisor (Gillman et al., 2000). Asimismo, tienen un $15 \%$ menos de riesgo de sobrepeso (Taveras et al., 2005) y una menor probabilidad de saltarse el desayuno (Videon y Manning, 2003). Finalmente, se ha comprobado que los niños que participan en programas de huertos escolares mejoran sus conocimientos sobre la alimentación y sobre las actividades de cultivo, mostrando más curiosidad por probar nuevos sabores (Chauliac et al., 1996) y una mejor disposición al sabor de las verduras (Morgan et al., 2010).

\section{CARACTERIZACIÓN DE LA COMPETENCIA EN ALIMENTACIÓN}

En la actualidad existen, en el contexto de la alimentación, una serie de conceptos emergentes que se vienen usando cada vez más en las políticas de salud, en la investigación y en la educación, para caracterizar a un ciudadano bien formado. Todos ellos son bastante similares, en líneas generales, con respecto a los conocimientos, habilidades, actitudes y valores que consideran necesarios para que una persona pueda llevar a cabo una alimentación saludable, si bien difieren en algunos aspectos concretos y, fundamentalmente, en los términos que utilizan para su denominación.

Nos referimos en concreto a los denominados alfabetización en alimentación (food literacy) (Cullerton, Vidgen y Gallegos, 2012), habilidades básicas en alimentación (food skills) (Vanderkooy, 2010) y competencias en alimentación (food competences) (Food Standards Agency, 2007 y 2009). En las tablas 1,2 y 3 se presentan, a través de ejemplos de los propios autores, los contenidos que dan a cada uno de los dominios en que subdividen cada concepto. 
La Food Standards Agency (FSA, 2007 y 2009) del Reino Unido desarrolló, a partir de un consenso entre expertos, un marco de competencias en alimentación (food competences), que recoge las habilidades y los conocimientos esenciales sobre alimentación que los niños y jóvenes deben poseer, comprender y ser capaces de aplicar a fin de ayudarles a tomar decisiones más saludables que los beneficien ahora y en el futuro.

Para la FSA, este marco ayudará a establecer los elementos esenciales para que las escuelas y las organizaciones comunitarias puedan proporcionar a los jóvenes un conjunto coherente de conocimientos y destrezas sobre alimentación. Estas competencias se consideran progresivas y acumulativas, y se aplican a todas las experiencias de aprendizaje, por lo que pueden ser adquiridas en la escuela, en la casa o mediante otras actividades.

Estas competencias se organizan en torno a cuatro temas: tres centrados principalmente en los conocimientos (los alimentos, la seguridad y la salud de la dieta y la sensibilización como consumidores responsables) y otro en las habilidades (cocinar y manipular alimentos) (tabla 1).

Tabla 1.

Ejemplos de competencias incluidas en cada uno de los cuatro temas propuestos por la Food Standard Agency (2007 y 2009)

\begin{tabular}{|c|c|}
\hline \multicolumn{2}{|r|}{$\begin{array}{l}\text { Competencias en alimentación (food competences) } \\
\text { Food Standard Agency (2007 y 2009) }\end{array}$} \\
\hline Temas & Ejemplos de competencias \\
\hline $\begin{array}{l}\text { Los alimentos. } \\
\text { Dieta y salud }\end{array}$ & $\begin{array}{l}\text { Hacer caso de los consejos sobre una alimentación saludable para elegir una dieta variada y } \\
\text { equilibrada a sus necesidades, además de ser físicamente activo. } \\
\text { Saber que los alimentos aportan energía y nutrientes en cantidades diferentes, que tienen fun- } \\
\text { ciones importantes en el cuerpo y que las personas requieren cantidades diferentes durante } \\
\text { su vida. } \\
\text { Comprender la importancia del equilibrio energético y las consecuencias de excesos o deficien- } \\
\text { cias de la dieta. } \\
\text { Ser capaz de mantener un peso saludable a lo largo de la vida, comprendiendo la relación entre } \\
\text { dieta y actividad física. }\end{array}$ \\
\hline $\begin{array}{l}\text { La seguridad } \\
\text { y la salud de la dieta }\end{array}$ & $\begin{array}{l}\text { Saber que la seguridad alimentaria significa prevenir la contaminación, el deterioro y la descom- } \\
\text { posición durante el manejo y el almacenamiento de los alimentos. } \\
\text { Comprender y desarrollar buenas prácticas de seguridad alimentaria. } \\
\text { Utilizar la información de las etiquetas de los alimentos para almacenarlos correctamente. }\end{array}$ \\
\hline $\begin{array}{l}\text { La sensibilización como } \\
\text { consumidores responsa- } \\
\text { bles. Conciencia como } \\
\text { consumidor }\end{array}$ & $\begin{array}{l}\text { Investigar dónde y cómo se producen y se venden los alimentos. } \\
\text { Hacer uso de la información de las etiquetas de los alimentos para ayudarse a tomar una deci- } \\
\text { sión sobre su compra. } \\
\text { Saber que los alimentos se producen, se procesan y se venden de diferentes maneras, como la } \\
\text { agricultura convencional y la ecológica, el comercio justo, etc. } \\
\text { Comparar el coste de los alimentos al comer fuera o al cocinar en casa. } \\
\text { Comprender la influencia de la comercialización de alimentos, la publicidad y la promoción } \\
\text { sobre la propia dieta y el propio comportamiento de compra. } \\
\text { Tener en cuenta una serie de factores al elegir los alimentos (estacionalidad, comida local, sos- } \\
\text { tenibilidad, etc.). }\end{array}$ \\
\hline $\begin{array}{l}\text { Cocinar y manipular } \\
\text { alimentos }\end{array}$ & $\begin{array}{l}\text { Utilizar una amplia gama de técnicas de preparación y métodos de cocinado, por ejemplo, freír, } \\
\text { cocer al vapor o mezclar. } \\
\text { Ser capaz de modificar las recetas y platos para hacerlas más saludables, usando distintos méto- } \\
\text { dos de cocina y/o cambiando los ingredientes. } \\
\text { Minimizar los residuos de alimentos y reciclar los envases. }\end{array}$ \\
\hline
\end{tabular}


Como parte del "Plan de vida activa y alimentación saludable", el Ministerio de Promoción de la Salud de la provincia canadiense de Ontario editó un documento guía donde se definían las habilidades básicas en alimentación (food skills). A un nivel individual y familiar, las habilidades básicas en alimentación son un conjunto, complejo, interrelacionado y centrado en la persona, y son necesarias para proveer y preparar alimentos seguros, nutritivos y culturalmente aceptables para todos los miembros de la familia (Vanderkooy, 2010). Estas habilidades (tabla 2), descritas para un contexto familiar, implican conocimientos, planificación, conceptualización, manipulación y percepción de los alimentos.

Tabla 2.

Definiciones operativas sobre los contenidos

de cada una de las destrezas básicas en alimentación propuestas por Vanderkooy (2010)

\begin{tabular}{|c|c|}
\hline \multicolumn{2}{|r|}{$\begin{array}{l}\text { Técnicas o destrezas básicas en alimentación (food skills) } \\
\text { Vanderkooy (2010) }\end{array}$} \\
\hline Destrezas básicas & Definiciones operativas \\
\hline El conocimiento & $\begin{array}{l}\text { Sobre los alimentos. Conocer las variedades de alimentos existentes y los ingredientes de las } \\
\text { comidas. } \\
\text { Nutricional. Comprender qué nutrientes se necesitan para mantener una vida sana y dónde } \\
\text { encontrarlos. } \\
\text { Sobre lectura de las etiquetas. Saber cómo determinar el valor nutricional de los alimentos a } \\
\text { partir de la lectura de las etiquetas. } \\
\text { Sobre la seguridad alimentaria. Comprender las normas básicas de seguridad para el almace- } \\
\text { namiento, la preparación y la manipulación de los alimentos. } \\
\text { Sobre la sustitución de ingredientes. Conocer la variedad de alimentos que se pueden utilizar } \\
\text { para lograr una alimentación saludable y comprender qué alimentos se pueden intercambiar } \\
\text { dentro de una receta dada sin cambiar su valor nutricional. }\end{array}$ \\
\hline La planificación & $\begin{array}{l}\text { Comprensión de la forma correcta en que se pueden organizar las comidas para que ofrezcan } \\
\text { un valor nutricional óptimo. } \\
\text { Capacidad para comprar y preparar los alimentos siguiendo un presupuesto. } \\
\text { Comprensión de lo que se requiere para preparar las comidas, incluyendo cuánto tiempo y } \\
\text { qué tareas son necesarias. } \\
\text { Capacidad para enseñar a los niños estas habilidades, para que sean capaces de tomar decisio- } \\
\text { nes correctas sobre los alimentos durante toda su vida. }\end{array}$ \\
\hline $\begin{array}{l}\text { La conceptualización de } \\
\text { los alimentos }\end{array}$ & $\begin{array}{l}\text { Capacidad y conocimiento para idear comidas desde los ingredientes primarios o a partir de } \\
\text { los restos de otras comidas. } \\
\text { Capacidad para ajustar recetas conocidas para adaptarse a las necesidades de cada momento. }\end{array}$ \\
\hline $\begin{array}{l}\text { Las técnicas mecánicas } \\
\text { para preparar comidas }\end{array}$ & $\begin{array}{l}\text { Conocimiento de las técnicas para preparar comidas de forma segura y creativa: picar, mezclar, } \\
\text { cocinar de distintas formas, seguir recetas. }\end{array}$ \\
\hline $\begin{array}{l}\text { La percepción sensorial de } \\
\text { los alimentos }\end{array}$ & $\begin{array}{l}\text { Capacidad para usar los sentidos cuando se cocinan los alimentos a fin de apreciar la textura, } \\
\text { el sabor, el aroma, etc. }\end{array}$ \\
\hline
\end{tabular}

Finalmente, Vidgen y Gallegos (2011) coordinaron una consulta entre expertos de la que surgió la siguiente definición de alfabetización en alimentación (food literacy) para el Gobierno del Estado australiano de Queensland: la capacidad relativa para comprender básicamente la naturaleza de los alimentos y la importancia que tienen para la persona, así como la manera en que esta puede obtener información acerca de sus alimentos, procesarla, analizarla y actuar en consecuencia.

Del estudio también emergió un modelo para describir esta alfabetización (tabla 3), que incluye ocho dominios: el acceso, la comida, la planificación y gestión, la selección, la procedencia de los alimentos, la preparación, la nutrición y el lenguaje sobre el tema (Cullerton, Vidgen, y Gallegos, 2012). De todas formas, las autoras subrayan que lo que la gente necesita saber y entender acerca de los ali- 
mentos para utilizarlo con el fin de satisfacer sus necesidades parece ser contextual en gran medida, en lugar de ser un conjunto universal de competencias que se puedan aplicar en todos los entornos.

Tabla 3.

Algunos de los componentes incluidos en cada uno

de los dominios de la alfabetización en alimentación (Cullerton, Vidgen y Gallegos, 2012)

\begin{tabular}{|c|c|}
\hline \multicolumn{2}{|r|}{$\begin{array}{l}\text { Alfabetización en alimentación (food literacy) } \\
\text { Cullerton, Vidgen y Gallegos (2012) }\end{array}$} \\
\hline Dominios & Algunos componentes \\
\hline Acceso & $\begin{array}{l}\text { Saber cómo tener acceso a los alimentos. } \\
\text { Ser crítico con el sistema de suministro de alimentos y ser capaz de proponer mejoras. }\end{array}$ \\
\hline Comida & $\begin{array}{l}\text { Ser capaz de participar y sentarse a comer de una manera social, tratando de favorecer la posibilidad } \\
\text { de formar parte de un grupo. } \\
\text { Estar dispuesto a probar comidas desconocidas. }\end{array}$ \\
\hline $\begin{array}{l}\text { Planificación } \\
\text { y gestión }\end{array}$ & $\begin{array}{l}\text { Ser capaz de planificar la compra de alimentos para cubrir las necesidades nutritivas. } \\
\text { Ser capaz de ajustar las compras al dinero disponible. }\end{array}$ \\
\hline Selección & $\begin{array}{l}\text { Ser capaz de juzgar críticamente las formas de producción y envasado de los alimentos, así como sus } \\
\text { anuncios, promociones y marketing. } \\
\text { Ser capaz de leer las etiquetas de los alimentos comprendiendo la lista de ingredientes, la información } \\
\text { nutricional y las columnas de ingesta por } 100 \mathrm{~g} \text { y por ración. }\end{array}$ \\
\hline $\begin{array}{l}\text { Procedencia } \\
\text { de los alimentos }\end{array}$ & $\begin{array}{l}\text { Saber plantar, hacer crecer y cosechar algunos alimentos. } \\
\text { Conocer el origen de los alimentos y ser consciente del contexto político, ecológico y social en que se } \\
\text { produjeron. }\end{array}$ \\
\hline Preparación & $\begin{array}{l}\text { Conocer la forma de preparar alimentos de manera atractiva y comestible. } \\
\text { Saber hacer de cuatro a seis comidas, incluyendo una de cada uno de los grupos de alimentos. } \\
\text { Saber cómo seguir una receta, incluso si consta de cuatro o cinco pasos. } \\
\text { Conocer y aplicar las normas de higiene y seguridad de los alimentos. Saber cómo almacenar los } \\
\text { alimentos. } \\
\text { Saber cómo eliminar los desechos de forma respetuosa con el medio ambiente. }\end{array}$ \\
\hline Nutrición & $\begin{array}{l}\text { Comprender las guías alimentarias y cómo traducir sus consejos en alimentos y hábitos alimentarios. } \\
\text { Saber clasificar los alimentos, según su composición, en los grupos de alimentos. } \\
\text { Saber cuáles son los componentes de una dieta básica saludable. Ser consciente del papel de los dife- } \\
\text { rentes nutrientes y conocer las necesidades diarias de cada uno. } \\
\text { Saber que todos los alimentos son buenos y solo las cantidades ingeridas pueden ser desaconsejables. } \\
\text { Tener idea sobre las porciones y la frecuencia de ingesta. }\end{array}$ \\
\hline Lenguaje & $\begin{array}{l}\text { Conocer la terminología propia de los alimentos para ser capaz de hablar sobre ellos, seguir recetas, } \\
\text { leer etiquetas, etc. }\end{array}$ \\
\hline
\end{tabular}

Consideramos que el conjunto de estos tres estudios proporciona una caracterización bastante completa de los conocimientos, habilidades, actitudes y valores que son necesarios para que una persona pueda llevar a cabo una alimentación saludable.

En cuanto a su denominación, optamos por la de competencia en alimentación, ya que se corresponde más exactamente con los referentes curriculares que se han adoptado en nuestro país para la formación básica de los ciudadanos (MEC, 2006). Por lo tanto, en adelante se utilizará esta denominación para referirnos a la capacidad de una persona para alimentarse de forma saludable durante su vida, lo que implica seleccionar correctamente los alimentos que han de configurar su dieta y prepararlos de manera segura para su ingestión. 


\section{MARCO DE REFERENCIA PARA LA EDUCACIÓN OBLIGATORIA}

Una vez caracterizada la competencia en alimentación, la siguiente cuestión que habría que plantearse es la siguiente: ¿qué deberían aprender los niños y adolescentes, en la educación obligatoria, para que lleguen a ser capaces de desarrollar hábitos y estilos de vida saludables con respecto a su alimentación; es decir, para ser competentes en este contexto?

Teniendo en cuenta lo expuesto anteriormente, se presenta un marco de referencia de la competencia en alimentación para su desarrollo en la educación obligatoria, organizado en torno a siete dimensiones:

1. Los alimentos

2. Funcionamiento del cuerpo con respecto a la nutrición

3. Cocinar

4. Cultivar y elaborar alimentos

5. Comprar alimentos

6. Comer en compañía

7. La actividad física y el descanso

Estas siete dimensiones integran los conocimientos, capacidades, habilidades, actitudes y valores concretos que aparecen en los tres estudios analizados en el apartado anterior y que se han tomado como referencia. Todas ellas, en su conjunto, implican el desarrollo de la noción de alimentación saludable y la adquisición de hábitos saludables por parte de los niños y jóvenes.

La competencia en alimentación se desarrollará en la medida en que los niños y los adolescentes progresen en el conjunto de estas dimensiones. No obstante, podemos considerar que cuatro de ellas son primarias, en el sentido de que son las que más favorecen el desarrollo de la competencia. Se trata de las relativas a los alimentos, al funcionamiento del cuerpo con respecto a la nutrición, a cocinar y a la actividad física y el descanso.

A continuación se describen cada una de estas dimensiones.

\section{Los alimentos}

Se incluyen en esta dimensión los conocimientos científicos que llevan a la noción de alimentación equilibrada o saludable. Desde hace casi un siglo, las autoridades sanitarias y las sociedades científicas han venido difundiendo estos conocimientos a través de las clasificaciones de alimentos y las guías alimentarias (Aranceta y Serra, 2001). Las clasificaciones suelen representarse por medio de un gráfico, generalmente un diagrama en rueda o en pirámide, y se basan en su composición en nutrientes. Por lo tanto, entender esta información implica conocer el valor nutritivo de los alimentos más frecuentes en la dieta.

Además de manejar las guías alimentarias, traducir sus consejos en hábitos alimentarios y estar dispuestos a seguirlos, esta dimensión incluye la capacidad de leer las etiquetas de los alimentos, comprendiendo la lista de ingredientes y la información nutricional, y tener una idea correcta de los tamaños y pesos de las raciones a que se refieren, tanto las recomendaciones como las etiquetas de los alimentos (Seligson, 2003). 


\section{Funcionamiento del cuerpo con respecto a la nutrición}

Esta dimensión hace referencia al conocimiento científico sobre los procesos fisiológicos vinculados a la nutrición y sobre la forma en que los hábitos alimentarios pueden afectar a la salud. Se incluye aquí también: entender que las personas requieren cantidades diferentes de nutrientes y de energía a lo largo de su vida (por ejemplo, en el embarazo o en la infancia), y comprender la importancia del mantenimiento de un peso saludable y unos hábitos alimentarios regulares, ligados a la tradición cultural y al medio geográfico.

\section{Cocinar}

El conocimiento sobre los alimentos y la nutrición, al que aluden las dos primeras dimensiones, puede ser visto como "el saber qué comer", mientras que el conocimiento culinario sería "el saber cómo hacerlo" (Ternier, 2010). Asistimos en la actualidad a una "transición culinaria", un proceso en el que culturas enteras experimentan cambios fundamentales en la estructura y tipo de habilidades necesarias para preparar su comida cada día (Lang y Caraher, 2001). Por ello, adquirir las destrezas culinarias básicas se considera una competencia clave y una destreza vital en las escuelas primarias y secundarias (Mac Con Iomaire y Lydon, 2011).

Se incluye en esta dimensión la adquisición de las habilidades para cocinar los alimentos de manera atractiva y comestible, en el tiempo disponible y minimizando los desperdicios. Abarcaría saber hacer, al menos, de cuatro a seis comidas diferentes, una de cada uno de los grupos de alimentos y empleando diferentes métodos de cocinado, por ejemplo, al horno, freír, cocer al vapor, etc. Esto implicaría saber picar, mezclar, etc., utilizando los sentidos (para apreciar la textura o el sabor) y ser capaz de seguir recetas, ajustándolas a otras condiciones si fuese necesario. Asimismo, incluiría la capacidad de reconocer los ingredientes principales de un alimento procesado o cocinado, hacer un uso creativo de las sobras y saber cómo eliminar los desechos de forma respetuosa con el medio ambiente, reciclando los envases.

Por último, también se engloban en esta dimensión los aspectos relativos a la seguridad alimentaria, es decir, conocer y aplicar las normas de higiene que permitan prevenir la contaminación, el deterioro y la descomposición durante el manejo y el almacenamiento de los alimentos, de forma que estos sean seguros para comer.

\section{Cultivar y elaborar alimentos}

Esta dimensión alude al conocimiento de las formas más sencillas de cultivar alimentos, y de algunas técnicas elementales de elaboración de algunos de ellos. Por lo tanto, se refiere a saber plantar, hacer crecer y cosechar algunas frutas y hortalizas y conocer dónde y cómo se producen y se venden otros alimentos, por ejemplo, visitando una granja. También incluiría conocer procedimientos básicos de elaboración de algunos de ellos, como fabricar mermeladas, yogures, vinagres, etc. La experiencia de cultivar alimentos puede facilitar la valoración de una serie de factores, como la estacionalidad, el origen y las condiciones en que han sido producidos (contexto político, ecológico y social, mediante agricultura convencional o ecológica, comercio justo, etc.).

\section{Comprar alimentos}

Esta dimensión se refiere a ser capaz de presupuestar, comprar y preparar los alimentos a partir de diferentes situaciones y de valorar la importancia de estas tareas. Asimismo, incluye: comprender la influencia de la comercialización, la publicidad y el marketing sobre la propia dieta y el propio com- 
portamiento de compra, juzgando críticamente el sistema de producción y envasado de los alimentos; comparar el coste de los alimentos en diferentes circunstancias, por ejemplo, al comer fuera o al cocinar en casa y, finalmente, ser capaz de utilizar la información de las etiquetas de los alimentos para ayudarse a tomar una decisión de compra o para almacenarlos correctamente. La preparación de comidas ajustadas a un presupuesto para equilibrar las necesidades de nutrición con las preferencias alimentarias y los horarios de los miembros de la familia es una tarea esencial entre las relacionadas con la alimentación (Chenhall, 2010).

\section{Comer en compañía}

Se refiere a valorar el hecho de compartir la comida con la familia o los compañeros, en vez de hacerlo de manera individual mientras se realiza otra actividad, generalmente frente a pantallas. Incluye ser capaz de participar y sentarse a comer de una manera social, favoreciendo la posibilidad de formar parte de un grupo, así como estar dispuesto a probar nuevos alimentos. Numerosas investigaciones han coincidido en que promocionar las comidas en grupo mejoraría la calidad de la dieta, reduciría el exceso de peso y mejoraría los resultados educativos y sociales (Videon y Manning, 2003; Fulkerson et al., 2006).

\section{La actividad física y el descanso}

Esta dimensión alude a la adquisición de hábitos adecuados de descanso y de actividad física a lo largo de toda la vida. Incluiría ser físicamente activo y comprender la relación entre dieta y actividad física para mantener un peso saludable a lo largo de la vida, al mismo tiempo que entender la necesidad del descanso adecuado como complemento imprescindible de las actividades físicas y mentales. La reducción de los factores de riesgo relacionados con las ENT mediante una adecuada actividad física ya se recomendó en la Estrategia mundial sobre régimen alimentario, actividad física y salud (oMs/wHO, 2004).

En la tabla 4 se recogen las siete dimensiones que configuran la competencia en alimentación en la educación obligatoria y ejemplos de actividades que ilustran cómo se podría abordar cada una de ellas.

Tabla 4.

Dimensiones de la competencia en alimentación en la educación obligatoria y ejemplos de actividades para desarrollarlas

\begin{tabular}{|l|l|}
\hline Dimensión de la competencia & \multicolumn{1}{|c|}{ Ejemplos de actividades } \\
\hline 1. Los alimentos & $\begin{array}{l}\text { Encuesta de ingesta durante, al menos, una semana. } \\
\text { Simulación de una dieta virtual a través del ordenador. }\end{array}$ \\
\hline $\begin{array}{l}\text { 2. El funcionamiento del } \\
\text { cuerpo con respecto a la } \\
\text { nutrición }\end{array}$ & $\begin{array}{l}\text { Valoración del estado nutricional: medida del IMC, de la tensión arterial, del número de } \\
\text { latidos cardiacos por minuto... } \\
\text { Lectura de libros o de páginas web que expliquen la etiología de algunas patologías o } \\
\text { trastornos relacionados con la alimentación. }\end{array}$ \\
\hline 3. Cocinar & $\begin{array}{l}\text { Clases de cocina. } \\
\text { Cocinar en casa. }\end{array}$ \\
\hline $\begin{array}{l}\text { 4. Cultivar y elaborar los } \\
\text { alimentos }\end{array}$ & $\begin{array}{l}\text { Participación en huertos escolares. } \\
\text { Elaboración de alimentos. }\end{array}$ \\
\hline
\end{tabular}




\begin{tabular}{|l|l|}
\hline Dimensión de la competencia & \multicolumn{1}{|c|}{ Ejemplos de actividades } \\
\hline 5. Comprar alimentos & $\begin{array}{l}\text { Visitas a mercados y supermercados con un presupuesto y una serie de comidas para } \\
\text { preparar. } \\
\text { Jurados de degustación. } \\
\text { Lectura comparativa de etiquetas de diferentes marcas. } \\
\text { Análisis comparativo de productos de varias marcas con la ayuda de revistas de organi- } \\
\text { zaciones de consumidores. } \\
\text { Análisis de publicidad de productos alimenticios (de imagen fija o en movimiento). } \\
\text { Lectura de recetas de comidas. }\end{array}$ \\
\hline 6. Comer en compañía & $\begin{array}{l}\text { Participación en comidas en grupo en la escuela (en el comedor, en las actividades ex- } \\
\text { traescolares, etc.). }\end{array}$ \\
\hline $\begin{array}{l}\text { 7. La actividad física y el des- } \\
\text { canso }\end{array}$ & $\begin{array}{l}\text { Participación en equipos deportivos, grupos de baile, senderismo, etc. } \\
\text { Acceso a la escuela a pie o en bicicleta de forma regular. }\end{array}$ \\
\hline
\end{tabular}

Desarrollar en la práctica educativa las dimensiones descritas de la competencia en alimentación implicaría, entre otros aspectos, un sistema más flexible de organización del trabajo escolar, de tal forma que, por ejemplo, la realización de programas, módulos o proyectos interdisciplinares pudieran coexistir con su enseñanza desde materias o asignaturas concretas.

\section{CONSIDERACIONES FINALES}

En este artículo se ha presentado un marco de referencia para el desarrollo de la competencia en alimentación en la educación obligatoria basado en estudios actuales sobre la situación en este contexto y sobre los conocimientos, habilidades, actitudes y valores necesarios para que una persona pueda llevar a cabo una alimentación saludable.

El procedimiento de trabajo aquí desarrollado para caracterizar esta competencia, que es solo uno entre los que se pueden seguir, ha intentado en todo momento tener presente que la finalidad de las competencias básicas es capacitar a las personas a tomar decisiones responsables en las diferentes facetas, personales, sociales y profesionales, de sus vidas (Perrenoud, 2012).

Este marco de referencia puede ser de utilidad para el diseño y la aplicación de propuestas curriculares que tengan como finalidad que los escolares, a los 16 ańos, como ciudadanos, puedan tomar decisiones responsables en el ámbito de su alimentación.

Finalmente, consideramos que el trabajo presentado puede continuarse en dos direcciones. Por un lado, profundizando en el campo del aprendizaje de la alimentación, perfilando y matizando mejor sus múltiples aspectos (Cabello, Blanco y España, 2009). Esto ayudaría a fundamentar las tareas relativas a delimitación y secuenciación de contenidos, así como a la selección de objetos de estudios. Por otro lado, diseñando, llevando a la práctica y evaluando propuestas didácticas en diferentes etapas y ciclos de la educación obligatoria a partir de este marco de referencia.

\section{AGRADECIMIENTOS}

Este artículo forma parte del proyecto de I+D+i Diseño y evaluación de un modelo para el fomento de la competencia científica en la educación obligatoria (10-16 años) (EDU 2009-07173), financiado por el Ministerio de Ciencia e Innovación en la convocatoria de 2009.

Los autores desean mostrar su agradecimiento a los revisores y editores por sus comentarios y sugerencias, lo que ha permitido, sin duda, precisar el enfoque y el contenido del artículo. 


\section{REFERENCIAS BIBLIOGRÁFICAS}

Aranceta, J. y Serra L. (2001). Estructura general de las Guías alimentarias para la población española. Decálogo para una dieta saludable. En senc. Guias Alimentarias para la Población Española: recomendaciones para una dieta saludable. Madrid: Sociedad Española de Nutrición Comunitaria, pp. 183-194.

Banegas, J.; Graciani, A.; Guallar-Castillón, P.; León-Muñoz, L.; Gutiérrez-Fisac, J.; LópezGarcía, E.; Otero-Rodríguez, A.; Regidor, E.; Taboada, J.; Aguilera, M.; Villar, F.; Zuluaga, M. y Rodríguez-Artalejo, F. (2011). Estudio de Nutrición y Riesgo Cardiovascular en España (Enrica). Madrid: Departamento de Medicina Preventiva y Salud Pública. Universidad Autónoma de Madrid.

Banet, E. (2001). Los procesos de nutrición humana. Madrid: Síntesis Educación.

BANET, E. (2010). Finalidades de la educación científica en educación secundaria: aportaciones de la investigación educativa y opinión de los profesores. Enseñanza de las Ciencias, 28(2), pp. 199-214.

Banet, E. y NúŃEz, F. (1992). La digestión de los alimentos: un plan de actuación en el aula fundamentado en una secuencia constructivista del aprendizaje. Enseñanza de las Ciencias, 10(2), pp. 139-147.

Blanco, A.; España, E. y González, F. J. (2010). Un proyecto de investigación para el fomento de la competencia científica en la educación obligatoria. En A. Quesada y A. Abril (eds.). Actas de los XXIV Encuentros de Didáctica de las Ciencias Experimentales, pp. 729-735.

Bybee, R. (1997). Achieving scientific literacy: from purposes to practices. Portsmouth: Heineeman.

CaAmaño, A. (2005). Presentación de la monografía: Contextualizar la ciencia. Una necesidad en el nuevo currículo de ciencias. Alambique, 46, pp. 5-8.

Cabello, A.; Blanco, A. y España, E. (2009). Una hipótesis de trabajo para investigar el progreso en la comprensión de la alimentación humana por parte de los alumnos. Enseñanza de las Ciencias. Número extra VIII Congreso, pp. 1729-1735.

Chamizo, J. e IzQuierdo, M. (2005). Ciencia en contexto: una reflexión desde la filosofía. Alambique, 46, pp. 9-17.

Chauliac, M.; Barros, T.; Masse-Raimbault, A. y Yepez, R. (1996). School gardens in the rural Andes. Food, Nutrition and Agriculture, 16, pp. 14-22.

Chenhall, C. (2010). Improving Cooking and Food Preparation Skills: A Synthesis of the Evidence to Inform Program and Policy Development. Government of Canada.. Disponible en: <http://www.hcsc.gc.ca/fn-an/alt_formats/pdf/nutrition/child-enfant/cfps-acc-synthes-eng.pdf>. (Última consulta: 18 de junio de 2013).

Cullerton, K.; Vidgen, H. y Gallegos, D. (2012). A review of food literacy interventions targeting disadvantaged young people. Disponible en: <http://eprints.qut.edu.au/53753/1/food_literacy_interventions_review_final.pdf>. (Última consulta: 4 de diciembre de 2012).

Da Silva, R.; Bach-Faig, A.; Raidó, B.; Buckland, G.; Vaz De Almeida, M. y Serra-Majem, L. (2009). Worldwide variation of adherence to the Mediterranean diet, in 1961-1965 and 20002003. Public Health Nutrition, 12(9A), pp. 1676-1684. http://dx.doi.org/10.1017/S1368980009990541

De Jong, O. (2006). Context-based chemical education: How to improve it? Paper based on the plenary lecture presented at the 19th ICCE, Seoul, Korea, 12-17 August. Disponible en: <http://old. iupac.org/publications/cei/vol8/0801xDeJong.pdf>. (Última consulta: 4 de diciembre de 2012).

Del Pozo, S.; García, V.; Cuadrado, C.; Ruiz, E.; Valero, T.; Ávila, J. y Varela Moreiras, G. (2012). Valoración Nutricional de la Dieta Española de acuerdo al Panel de Consumo Alimentario. Madrid: Fundación Española de la Nutrición (FEN). 
Drewnowski, A. y Popkin, B. (1997). The nutrition transition: new trends in the global diet. Nutrition Reviews, 55, pp. 31-43.

http://dx.doi.org/10.1111/j.1753-4887.1997.tb01593.x

Fensham, P. (2007). Competences, from within and without: new challenges and possibilities for scientific literacy. En C. Linder; L. Östman y P. Wickman (eds.). Promoting scientific literacy: science education research in transaction. Proceedings of the Linnaeus Tercentenary Symposium held at Uppsala University. Uppsala University: Sweden, pp. 113-119.

Fensham, P. (2009). Real world contexts in PISA science: implications for context-based science education. Journal of Research in Science Teaching, 46(8), pp. 884-896. http://dx.doi.org/10.1002/tea.20334

Fernández-SAn JuAn, P. M. (2006). Dietary habits and nutritional status of school aged children in Spain. Nutrición Hospitalaria, 21(3), pp. 374-378.

Fourez, G. (1997). Alfabetización cientifica y tecnológica. Acerca de las finalidades de la enseñanza de las ciencias. Buenos Aires: Colihue.

Food Standards Agency (FSA) (2007). Food Competency framework: food skills and knowledge for children and young people by age of 7-9, 11-12, 14 and 16+. Disponible en: <http://www.food. gov.uk/multimedia/pdfs/competencyria.pdf>. (Última consulta: 4 de diciembre de 2012).

Food Standards Agency (FSA) (2009). Users' guide. Secondary school aged materials (11 to 14 years and 16+ years). Disponible en: <http://www.food.gov.uk/multimedia/pdfs/foodrouteuser2.pdf>. (Última consulta: 4 de diciembre de 2012).

Fulkerson, J. A.; Neumark-Sztainer, D. y Story, M. (2006). Adolescent and Parent Views of Family Meals. Journal of the American Dietetic Association, 106, pp. 526-532. http://dx.doi.org/10.1016/j.jada.2006.01.006

García, S. y Martínez, M. C. (2009). La nutrición: una función vital que invita a la reflexión didáctica. Aula de Innovación Educativa, 183-184, pp. 37-40.

García-Closas, R.; Berenguer, A. y González, C. (2006). Changes in food supply in Mediterranean countries from 1961 to 2001. Public Health Nutrition, 9(1), pp. 53-60. http://dx.doi.org/10.1079/PHN2005757

Gargallo, M. (1998). Dieta Equilibrada. Los siete grupos de alimentos. En C. Vázquez; C. LópezNomdedeu y A. de Cos. Alimentación y nutrición: manual teórico-práctico. Madrid: Díaz de Santos, pp. $65-77$.

Gilbert, J. (2006). On the nature of "context" in chemical education. International Journal of Science Education, 28(9), pp. 957-976. http://dx.doi.org/10.1080/09500690600702470

Gillman, M.; Rifas-Shiman, S.; Frazier, A.; Rockett, H.; Camargo, C.; Field, A.; Berkey, C. y Colditz, G. (2000). Family dinner and diet quality among older children and adolescents. Archives of Family Medicine, 9(3), pp. 235-240.

http://dx.doi.org/10.1001/archfami.9.3.235

Gutiérrez, A. (2006). PISA y la evaluación de la alfabetización científica. Investigación en la Escuela, 60, pp. 65-77.

Hawkes, C. (2007). Globalization, Food and Nutrition Transitions. Commission on Social Determinants of Health. Globalization and Health Knowledge Network: Research Papers. WHO. Disponible en: <http://www.who.int/social_determinants/resources/gkn_hawkes.pdf>. (Última consulta: 4 de diciembre de 2012).

Hernández, F. (2006). El informe PISA: una oportunidad para replantear el sentido de aprender en la escuela secundaria. Revista de Educación, número extraordinario, pp. 357-379. 
Instituto de Información Sanitaria (irs) (2011). Encuesta Europea de Salud en España 2009: Principales resultados. Ministerio de Sanidad, Política Social e Igualdad. Disponible en: <www.msssi. gob.es/estadEstudios/estadisticas/EncuestaEuropea/Principales_Resultados_Informe.pdf>. (Última consulta: 4 de diciembre de 2012).

Instituto Nacional de Estadística (Ine) (2006). Encuesta Nacional de Salud de España. Ministerio de Sanidad, Servicios Sociales e Igualdad. Disponible en: <www.msps.es/estadEstudios/estadisticas/encuestaNacional/encuesta2006.htm>. (Última consulta: 18 de junio de 2013).

LANG, T. y CARAher, M. (2001). Is there a culinary skills transition? Data and debate from the UK about changes in cooking culture. Journal of the HEIA, 5(2), pp. 2-14.

Larson, N.; Perry, CH.; Story, M. y Neumark-Sztainer, D. (2006): Food Preparation by Young Adults Is Associated with Better Diet. Quality Journal of the American Dietetic Association, 106(12), pp. 2001-2007.

http://dx.doi.org/10.1016/j.jada.2006.09.008

Mac Con Iomaire, M. y Lydon, J. (2011). The Current State of Cooking in Ireland: The Relationship between Cooking Skills and Food Choice. Tourism and Hospitality Research in Ireland Conference (THRIC). Athlone Institute of Technology.

Marmot, M.; Smith, G., Stansfeld, S.; Patel, C.; North, F.; Head, J.; White I., Brunner E. y Feeney A. (1991). Health Inequalities among British civil servants: the Whitehall II study. Lancet, 337(8.754), pp. 1387-1393.

http://dx.doi.org/10.1016/0140-6736(91)93068-K

MEC (2006). Real Decreto 1631/2006, de 29 de diciembre, por el que se establecen las enseñanzas mínimas correspondientes a la Educación Secundaria Obligatoria. En BOE, n. ${ }^{\circ} 5$, de viernes 5 enero de 2007.

Membiela, P. y Cid, M. (1998). Desarrollo de una unidad didáctica centrada en la alimentación humana, social y culturalmente contextualizada. Enseñanza de las Ciencias, 16(3), pp. 499-511.

Merelles, T.; Costa, A.; Sánchez, A. y Ruano, L. (2005). La educación nutricional desde la Atención Primaria. En C. Vázquez; C. López-Nomdedeu y A. de Cos. Alimentación y nutrición: manual teórico-práctico. Madrid: Díaz de Santos, pp. 273-283.

Ministerio de SANidad (2011). Estudio ALADINO. Disponible en: <http://www.naos.aesan.msssi. gob.es/en/naos/investigacion/aladino/\#>. (Última consulta: 18 de junio de 2013).

Moreiras, O., Carbajal; Cabrera, L. y Cuadrado, C. (2010). Tablas de Composición de Alimentos. Pirámide Ediciones S.A.

Moreno, C.; Muñoz, V.; Pérez, P. y Sánchez-Queija, I. (2005). Los adolescentes españoles y su salud. Resumen del estudio "Health Behaviour in School Aged Children" (HBSC-2002). Madrid: Ministerio de Sanidad, Política Social e Igualdad.

Moreno, C.; Muñoz, V.; Pérez, P.; Sánchez-Queija, I; Granado, M.; Ramos, P. y Rivera, F. (2008). Desarrollo adolescente y salud. Resultados del Estudio HBSC-2006 con chicos y chicas españoles de 11 a 17 años. Madrid: Ministerio de Sanidad y Consumo. Disponible en: <http://www.msc. es/profesionales/saludPublica/prevPromocion/promocion/saludJovenes/estudioHBSC/nacional_ hbsc.htm>. (Última consulta: 18 de junio de 2013).

Morgan, P.; Warren, J.; Lubans, D.; Saunders, K.; Quick, G. y Collins, C. (2010): The impact of nutrition education with and without a school garden on knowledge, vegetable intake and preferences and quality of school life among primary-school students. Public Health Nutrition, 13(11), pp. 1931-1940.

http://dx.doi.org/10.1017/S1368980010000959

oCDE (2006). PISA 2006. Marco de la Evaluación. Conocimientos y habilidades en Ciencias, Matemáticas y Lectura. Madrid: Santillana. 
OMs/who (2004). Global Strategy on Diet, Physical Activity and Health / Estrategia mundial sobre régimen alimentario, actividad física y salud. 57. a Asamblea mundial de la salud. Geneve: WHO; 2004. Disponible en: <http://www.who.int/dietphysicalactivity/goals/en/index.html>. (Última consulta: 4 de diciembre de 2012).

oms/who (2012). Estadísticas Sanitarias Mundiales 2012. Disponible en: <http://www.who.int/gho/ publications/world_health_statistics/ES_WHS2012_Full.pdf>. (Última consulta: 4 de diciembre de 2012).

Ortega, F.; Ruiz, J.; Castillo M.; Moreno L.; González-Gross M.; Warnberg, J. et al. (2005). Bajo nivel de forma física en los adolescentes españoles. Importancia para la salud cardiovascular futura (Estudio AVEna). Revista Española de Cardiología, 58, pp. 898-909.

http://dx.doi.org/10.1157/13078126

Ortega, R.; López-Sobaler, A.; Ballesteros, J.; Pérez-Farinós, N.; Rodríguez-Rodríguez, E.; Aparicio, A.; Perea, J. y Andrés, P. (2011). Estimation of salt intake by 24 h urinary sodium excretion in a representative sample of Spanish adults. British Journal of Nutrition, 105(5), pp. 787-794. http://dx.doi.org/10.1017/S000711451000423X

Patel, S. y Hu, F. (2008). Short Sleep Duration and Weight Gain: A Systematic Review. Obesity, 16(3), pp. 643-653.

http://dx.doi.org/10.1038/oby.2007.118

Pérez Gómez, A. (2008). ¿Competencias o pensamiento práctico? La construcción de los significados de representación y de acción. En J. Gimeno (comp.). Educar por competencias, ¿qué hay de nuevo? Madrid: Morata, pp. 88-95.

Perrenoud, P. (2012). Cuando la escuela pretende preparar para la vida ¿Desarrollar competencias o enseñar otros saberes? Barcelona: Graó.

Pinto, J. A. y Carbajal, A. (2006). Nutrición y salud. La dieta equilibrada, prudente o saludable. Servicio de Promoción de la Salud. Instituto de Salud Pública. Consejería de Sanidad de la Comunidad de Madrid. Disponible en: <www.madrid.org/sanidad>. (Última consulta: 18 de junio de 2013).

Polanco, I.; Tormo, R.; Guayta, R.; Costa, X. y Serra J. (2007). Hablemos del estreñimiento infantil. Barcelona: ACV Ediciones.

Pro, A. y Rodríguez, J. (2010). Aprender competencias en una propuesta para la enseñanza de los circuitos eléctricos en la educación primaria. Enseñanza de las Ciencias, 28(3), pp. 385-404.

Pro, A. (2012). Los ciudadanos necesitan conocimientos de ciencias para dar respuestas a los problemas de su contexto. En E. Pedrinaci (coord.). 11 ideas clave. El desarrollo de la competencia cientifica. Barcelona: Graó, pp. 83-104.

Ramos, P. (2009). Estilos de vida y salud en la adolescencia. Tesis doctoral. Sevilla: Universidad de Sevilla.

Salvador, G. y Serra, L. (1993). Grupos de Alimentos. En VV. AA. Nutrición y Dietética. Aspectos sanitarios. Plan de Educación Nutricional por el Farmacéutico PLENUFAR. Girona: Consejo General de Colegios Oficiales de Farmacéuticos, pp. 287-323.

Sanmarti, N.; Burgoa, B. y Nuño, T. (2011). ¿Por qué el alumnado tiene dificultad para utilizar sus conocimientos científicos escolares en situaciones cotidianas? Alambique, 67, pp. 62-69.

San Martín, H. (1989). Manual de Salud Pública y Medicina Preventiva. Barcelona: Masson.

Seligson, F. (2003). Serving Size Standards: Can They Be Harmonized? Nutrition Today, 38(6), pp. 247-253.

http://dx.doi.org/10.1097/00017285-200311000-00017

Serra, L. y Aranceta, J. (2000). Estudio enKid 1998-2000. Barcelona: Masson. 
Serra, L.; Ribas, L.; Ngo, J.; Aranceta, J.; Garaulet, M.; Carazo, E.; Mataix, J.; Pérez-RodriGo, C.; Quemada, M.; Tojo, R. y Vázquez, C. (2001). Risk of Inadequate Intakes of Vitamins $\mathrm{A}, \mathrm{B}_{1}, \mathrm{~B}, \mathrm{C}, \mathrm{E}$, Folate, Iron and Calcium in the Spanish Population Aged 4 to 18. International Journal for Vitamin and Nutrition Research, 71(6), 2001, pp. 325-331.

Serra, L.; Ribas, L.; Aranceta, J.; Pérez, C.; Saavedra y Peña (2003). Obesidad infantil y juvenil en España. Resultados del Estudio ENKID (1998-2000). Med Clin (Barc), 121(19), pp. 725-32. Disponible en: <http:/www.seedo.es/portals/seedo/consenso/Prevalencia_niños_Estudio_ENKID(Med_ Clin_2003).pdf >.

Short, F. (2003). Domestic cooking skills - what are they? Journal of the HEIA, 10(3), pp. 13-22.

Soliah, L.; Walter, J. y Antosh, D. (2006). Quantifying the impact of food preparation skills among college women. College Student Journal, 40(4), pp. 729-739.

Taveras, E.; Rifas-Shiman, S.; Berkey, C.; Rockett, H.; Field, A.; Frazier, A.; Colditz, G. y Gillman, M. (2005). Family dinner and adolescent overweight. Obesity Research, 13(5), pp. 900906.

http://dx.doi.org/10.1038/oby.2005.104

Ternier, S. (2010). Understanding and measuring cooking skills and knowledge as factors influencing convenience food purchases and consumption. Studies by Undergraduate Researchers at Guelph, 3(2), pp. 69-76.

Vanderkooy, P. (2010). Food skills of Waterloo Region adults. Fireside Chat Presentation. Disponible en: <http://www.chnet-works.ca/index.php?option=com_phocadownload\&view=category\&id=7 $\% 3$ Afireside-chat-presentations-2010\&Itemid=13\&lang=en\&limitstart=20>. (Última consulta: 4 de diciembre de 2012).

Varela Mosquera, G.; Moreiras, O. y Carbajal, A. (1988). Evolución del estado nutritivo y de los hábitos alimentarios de la población española. Serie "Divulgación”, n. ${ }^{\circ} 9$. Madrid: Fundación Española de la Nutrición.

Videon, T. y Manning, C. (2003). Influences on adolescent eating patterns: the importance of family meals. Journal Adolesc Health, 32, pp. 365-373. http://dx.doi.org/10.1016/S1054-139X(02)00711-5

Vidgen, H. y Gallegos, D. (2011). What is food literacy and does it influence what we eat: a study of Australian food experts. Queensland University of Technology. Disponible en: <http://eprints.qut. edu.au/45902/>. (Última consulta: 4 de diciembre de 2012).

WÄrnberg; Ruiz; Ortega; Romeo; González-Gross; Moreno; García-Fuentes; Gómez; Nova; Díaz; Marcos y Grupo avena (2006). Estudio avena (Alimentación y valoración del estado nutricional en adolescentes). Resultados obtenidos 2003-2006. Pediatría Integral, Supl., 1, 5 pp. 0-55.

Wilkinson, R. y Мавмот, M. (eds.) (2003). Social determinants of health: The Solid Facts. OMS. Disponible en: <http://www.euro.who.int/_data/assets/pdf_file/0005/98438/e81384.pdf> (Última consulta: 4 de diciembre de 2012).

Winkler, E. y Turrell, G. (2010). Confidence to cook vegetables and the buying habits of Australian households. Journal of the American Dietetic Association, 110(5), pp. 52-61. http://dx.doi.org/10.1016/j.jada.2010.03.007 


\title{
A framework for developing food competence in compulsory education
}

\author{
Enrique España Ramos \\ enrienri@uma.es \\ Aurelio Cabello Garrido \\ aacabellogarrido@gmail.com \\ Ángel Blanco López \\ ablancol@uma.es \\ Universidad de Málaga, Didáctica de las Ciencias Experimentales
}

In this paper, a theoretical framework for developing Food Competence in compulsory education is presented. The approaches involved initially lead us to step out of the school context and move into daily life in order to find out what the current situation in our society is. We begin with an updated review of studies on this subject, delimiting and defining what is meant by "context of human nutrition". Following this, the main areas for concern identified in Spanish children and young people regarding this context are analysed. Considering these aspects, the Food Competences that a citizen should have are also characterised in this paper.

Currently, there are several emerging concepts in the context of human nutrition which are increasingly used in health policy, research and education, and to characterise a well-trained citizen. Although they are quite similar in general, they differ in some specific aspects, particularly regarding the terms which are used to name them. We refer to the so-called 'food literacy' (Cullerton, Vidgen, \& Gallegos, 2012), 'food skills' (Vanderkooy, 2010) and 'food competences' (Food Standards Agency, 2007 and 2009). We consider that these three studies, analysed as a whole, provide a fairly complete characterisation of the knowledge, skills, attitudes and values that a person needs so as to follow a healthy diet throughout his/her life. In this paper, we propose using the term 'Food Competence' to refer to the ability of a person to eat healthily all his/her life. This implies the right selection of foods and their safe preparation for eating.

We then tackled the aspects of this competence that should be learned by children and adolescents throughout compulsory education. As a result, the framework for its development in compulsory education is described. It has been organised around seven axes:

- Food: Science-based knowledge leading to the notion of a balanced and healthy diet in order to turn the advice of the Dietary Guidelines into eating habits.

- The body's work regarding nutrition: Science-based knowledge on the physiological processes regarding nutrition and how eating habits can affect health.

- Cooking: The acquisition of basic cooking skills.

- Growing and processing food: The ability to cultivate some easy-to-grow vegetables and to use some simple methods of food preservation.

- Buying groceries: The ability to budget, buy and prepare food from different situations and to value the importance of these tasks.

- Eating with others: The ability to participate and to sit down to eat in a social way, valuing the importance of sharing food with others rather than eating watching a screen.

- Physical activity and rest: The acquisition of adequate rest habits and physical activity throughout life.

These seven axes include the knowledge, skills, abilities, attitudes and values that appear in the three studies taken as a reference. All of them, as a whole, contribute to the development of the concept of healthy eating and healthy habits among children and youth. Food Competence will occur to the extent that children and teens progress in all these axes. However, we think that four of them are of primary importance because they are the most beneficial to the development of the Food Competence. These are food, the functions of the body with respect to nutrition, cooking and physical activity and rest.

This framework may be useful for the design and implementation of curriculum proposals aimed to help schoolchildren make informed decisions in the field of food at the age of 16 and as citizens. Finally, this work can be continued in two directions. First, by studying human nutrition more thoroughly, i.e., profiling and better clarifying its many respects, which may help both to ground the delimitation and sequencing of content and to select objects of study. Second, this work could be continued through the design, implementation and assessment of educational proposals at the different stages of compulsory education. 
